\title{
Inter-Sector Beamforming with MMSE Receiver in the Downlink of TDD Cellular Systems
}

\author{
Jae-Heung Yeom and Yong-Hwan Lee
}

\begin{abstract}
The use of beamforming is effective for users in limited power environments. However, when it is applied to the downlink of a cellular system with universal frequency reuse, users near the sector boundary may experience significant interference from more than one sector. The use of a minimum mean square error (MMSE)-type receiver may not sufficiently cancel out the interference unless a sufficient number of receive antennas are used. In this paper, we consider the use of inter-sector beamforming that cooperates with a neighboring sector in the same cell to mitigate this interference problem in time-division duplex (TDD) environments. The proposed scheme can avoid interference from an adjacent sector in the same cell, while enhancing the transmit array gain by using the TDD reciprocity. The performance of the proposed scheme is analyzed in terms of the output signal-to-interference-plus-noise power ratio (SINR) and the output capacity when applied to an MMSE-type receiver. The beamforming mode can be analytically switched between the inter-sector and the single-sector mode based on the long-term channel information. Finally, the effectiveness of the proposed scheme is verified by computer simulation.
\end{abstract}

Index Terms: Array gain, beamforming, cooperation, interference, minimum mean square estimate (MMSE), sector boundary, time division duplex (TDD).

\section{INTRODUCTION}

Demand for higher throughput has motivated advanced wireless systems such as the third generation partnership long-term evolution (3GPP LTE) and mobile worldwide interoperability for microwave access (m-WiMAX) to employ multi-cell configuration with universal frequency reuse [1], [2]. It has been reported that the use of beamforming is effective in the downlink when users are power limited (i.e., at medium to low signalto-noise power ratio (SNR)) [3], [4]. In a time-division duplex (TDD) wireless system, base station (BS) can estimate channel state information (CSI) of the uplink using reference signals such as the channel sounding signal [5] and can utilize it for the transmit beamforming by means of channel response reciprocity [5], [6]. However, the use of beamforming with universal frequency reuse may cause users near the cell boundary to experience serious inter-cell interference [1], [2]. In particular, when the cell is divided into a number of sectors, users near the sector boundary may experience weak received signal strength (RSS) from the serving sector due to multi-sector antenna pattern as well as strong interference from adjacent sectors [6], [7].

Manuscript received December 16, 2007.

The authors are with the School of Electrical Engineering and INMC, Seoul National University, Seoul, Korea, email: jhyeom@ttl.snu.ac.kr, ylee@ snu.ac.kr.

This work was in part supported by the IT R\&D program of MKE/IITA (2008F-007-01, Intelligent Wireless Communication Systems in 3 Dimensional Environment).
Recently, advanced wireless systems consider the use of multiple receive antennas in the mobile station (MS) [2], [6], [8], enabling the use of a minimum mean square error (MMSE)type receiver to suppress interference, while reducing the fading effect [9]. However, when the number of strong interferers is larger than the number of receive antennas minus one, the output signal-to interference-plus-noise power ratio (SINR) of the MMSE receiver significantly deteriorates [10], [11].

To improve the performance of users near the sector/cell boundary, the use of BS coordination has recently been considered, where the BSs share the CSI and information streams to minimize the interference effect [12], [13]. However, this BS cooperation may incur so-called signaling overhead increasing in exponentially proportional to the number of coordinating BSs and suffer from performance degradation due to the signaling delay. A null beamformer has been recently introduced, where the interference to other co-channel cells is removed by exchanging the instantaneous inter-cell CSI [14]. However, it considers the use of a single receive antenna, neglecting the interference cancelation at the receiver. In practice, advanced wireless systems consider the use of real-time cooperation among sectors in the same cell, where the information between these sectors can be exchanged in real time without signaling overhead [8], [15].

On the other hand, the use of softer handover, macro diversity handover, and fast sector selection (FSS) with muting can be used to enhance the performance of users near the sector boundary without the exchange of inter-cell information [8], [16]. In the softer handover, both the serving sector and neighboring sector transmit the same data signal with different sector-specific scrambling codes, and the user softly combines the two signals before decoding. Note that the simultaneous transmission of same data signal may cause mutual interference. In the macrodiversity handover, the MS can demodulate signal without interfering each other, while obtaining radio frequency diversity. The performance depends on the code rate and the propagation delay between the sectors since it is the same as the cyclic shift transmit diversity [17]. In the FSS with muting, only one sector in better condition transmits data signal with doubled transmit power, while the other sector, called muted sector, does not transmit data signal to avoid mutual interference. However, these schemes do not consider the use of TDD reciprocity.

In this paper, to improve the performance of users near the sector boundary, we propose an inter-sector beamforming scheme that cooperates with an adjacent sector in the same cell. The proposed scheme can avoid interference from the adjacent sector in the same cell, while enhancing the transmit array gain using the TDD reciprocity. Moreover, it does not require additional signaling overhead compared to conventional beamform- 
ing (or single-sector beamforming) scheme. It is assumed that the MS transmits the reference signal using a single transmit antenna, as being considered in the IEEE 802.16e [2], [6] and IEEE 802.20 systems [18]. This implies that the BS should be able to generate a beamforming weight using partial knowledge of the CSI obtained through a single transmit antenna of the MS. The average output SINR and capacity of the MMSE receiver with the use of inter-sector beamforming are analyzed in the presence of correlation between the transmit antennas. According to the channel condition, the proposed scheme employs either single-sector or inter-sector beamforming to maximize the average capacity.

Following Introduction, Section II describes the system model in consideration. Section III presents the proposed intersector beamforming. The performance of the proposed scheme is analyzed in terms of the output SINR and the output capacity with combined the use of an MMSE-type receiver in Section IV. The performance of the proposed scheme is verified by computer simulation in Section V. Finally, conclusions are given in Section VI.

\section{SYSTEM MODEL}

Consider the downlink of a TDD cellular system that comprises $N$ hexagonal cells each of which comprises $S$ sectors (i.e., a total of $N S$ sectors). For ease of description, it is assumed that each sector uses $M$ transmit antennas and the MS uses two receive antennas. Assuming that the target user is serviced by sector $m$, its received signal can be represented as

$$
\begin{aligned}
\mathbf{y} & =\alpha_{m} \mathbf{H}_{m} \mathbf{w}_{m} x_{m}+\sum_{i \neq m} \alpha_{i} \mathbf{H}_{i} \mathbf{w}_{i} x_{i}+\mathbf{n} \\
& =\alpha_{m} \mathbf{H}_{m} \mathbf{w}_{m} x_{m}+\sum_{i \in \Omega, \neq m} \alpha_{i} \mathbf{H}_{i} \mathbf{w}_{i} x_{i}+\mathbf{z}
\end{aligned}
$$

where $x_{i}$ denotes the modulation symbol of sector $i$ with unit average power, $\alpha_{i}$ denotes the RSS from sector $i$ to the target user, $\mathbf{H}_{i}$ denotes the $(2 \times M)$ channel matrix from sector $i$ to the target user, $\mathbf{w}_{i}$ denotes the $(M \times 1)$ beam weight from sector $i$, $\mathbf{n}$ is the $(2 \times 1)$ additive white Gaussian noise (AWGN) vector, and $\Omega$ denotes an active set which comprises sectors with strong RSS, given by

$$
\Omega=\left\{k \mid \frac{\alpha_{k}^{2}}{\alpha_{m}^{2}} \geq \delta, 0 \leq k \leq N S-1\right\} .
$$

Here, $\delta$ denotes a threshold value for the choice of the active set and $\mathbf{z}$ denotes the $(2 \times 1)$ interference-plus-noise vector except the interference from sectors belonging to $\Omega$, which can be assumed to be zero-mean complex Gaussian with covariance $E\{\mathbf{z z}\}^{*}=N_{z} \mathbf{I}_{2}$ [19], where $\mathbf{I}_{2}$ denotes a $(2 \times 2)$ identity matrix. It is assumed that the BS receives the reference signal transmitted through the first antenna of the MS [2], [18]. Thus, it can be assumed that the BS generates the beam weight $\mathbf{w}_{m}$ based on the CSI estimated from the received reference signal. In addition, it is assumed that $\left\|\mathbf{w}_{m}\right\|^{2} \leq 1$, where $\|\cdot\|$ denotes the Frobenius norm [20].

\section{INTER-SECTOR BEAMFORMING}

Consider the use of inter-sector beamforming with cooperation between the sectors in the same cell to enhance the performance of users near the sector boundary. The inter-sector beamforming can extend from $(M \times 2)$ antenna configuration to $(2 M \times 2)$ by concatenating two adjacent sectors. It can allow the BS to receive the reference signal transmitted by users near the sector boundary without additional overhead compared to the single-sector beamforming. Thus, the BS can transmit the signal with beamforming using two sectors, while avoiding interference from the adjacent cooperating sector in the same cell. Assume that the BS transmits the target user signal through sectors $m$ and $m^{\prime}$. Then, the received signal can be represented as

$$
\begin{aligned}
\mathbf{y} & =\left[\begin{array}{cc}
\alpha_{m} \mathbf{H}_{m} \mathbf{w}_{m} & \alpha_{m^{\prime}} \mathbf{H}_{m^{\prime}} \mathbf{w}_{m^{\prime}}
\end{array}\right] x_{m}+\mathbf{z}_{t} \\
& =\alpha_{m}\left[\begin{array}{ll}
\mathbf{H}_{m} & \mathbf{H}_{m^{\prime}}
\end{array}\right] \boldsymbol{\Lambda}_{D} \mathbf{w}_{D} x_{m}+\mathbf{z}_{t} \\
& =\alpha_{m} \mathbf{H}_{D} \mathbf{w}_{D} x_{m}+\mathbf{z}_{t}
\end{aligned}
$$

where $\mathbf{z}_{t}=\sum_{i \in \Omega, \neq m, m^{\prime}} \alpha_{i} \mathbf{H}_{i} \mathbf{w}_{i} x_{i}+\mathbf{z}$, and $\mathbf{H}_{D}$ denotes the $(2 \times 2 M)$ channel matrix from sectors $m$ and $m^{\prime}$ to the target user, given by

$$
\mathbf{H}_{D}=\left[\begin{array}{ll}
\mathbf{H}_{m} & \mathbf{H}_{m^{\prime}}
\end{array}\right] \boldsymbol{\Lambda}_{D}
$$

$\mathbf{w}_{D}$ is a $(2 M \times 1)$ beamforming vector from sector $m$ and $m^{\prime}$ to the target user, and $\boldsymbol{\Lambda}_{D}$ denotes the normalized RSS matrix represented in a $(2 M \times 2 M)$ diagonal matrix whose first $M$ diagonal elements are one and the last $M$ ones are $\alpha_{m^{\prime}} / \alpha_{m}$. Note that the transmit power of $\mathbf{w}_{D}$ is given by

$$
\left\|\mathbf{w}_{D}\right\|^{2} \leq \max \left(\frac{\operatorname{tr}\left[\boldsymbol{\Lambda}_{D}^{2}\right]}{M}\right)=\max \left(1+\frac{\alpha_{m^{\prime}}^{2}}{\alpha_{m}^{2}}\right)
$$

where $\operatorname{tr}[\cdot]$ denotes the trace of a matrix. It can be seen that $\left\|\mathbf{w}_{D}\right\|^{2}=2$ when a neighboring sector with equal RSS is cooperated (i.e., $\alpha_{m}=\alpha_{m^{\prime}}$ ), and $\left\|\mathbf{w}_{D}\right\|^{2}=1$ when no sector is cooperated (i.e., $\alpha_{m^{\prime}}=0$ ).

When the channel is correlated, its covariance matrix can be represented as [21]

$$
\mathbf{R} \triangleq E\left\{\mathbf{H}_{D}^{*} \mathbf{H}_{D}\right\} / 2=\mathbf{Q} \boldsymbol{\Sigma}^{2} \mathbf{Q}
$$

where $E\{\cdot\}$ denotes the expectation, the superscript * denotes transpose conjugate, $\mathbf{Q}=\left[\begin{array}{lll}\mathbf{q}_{1} & \cdots & \mathbf{q}_{2 M}\end{array}\right]$ is a $(2 M \times 2 M)$ unitary matrix whose columns $\left\{\mathbf{q}_{k}\right\}$ are the eigenvectors of $\mathbf{R}$, and $\boldsymbol{\Sigma}^{2}$ is a $(2 M \times 2 M)$ diagonal matrix whose diagonal terms are descending-ordered eigenvalues (i.e., $\lambda_{1} \geq \cdots \geq \lambda_{2 M}$ ) of $\mathbf{R}$. The covariance matrix can be rewritten as

$$
\mathbf{R}=\boldsymbol{\Lambda}_{D}\left(E\left\{\left[\begin{array}{ll}
\mathbf{H}_{m} & \mathbf{H}_{m^{\prime}}
\end{array}\right]^{*}\left[\begin{array}{ll}
\mathbf{H}_{m} & \mathbf{H}_{m^{\prime}}
\end{array}\right] / 2\right\}\right) \boldsymbol{\Lambda}_{D}
$$

It can be seen that the RSS of two cooperating sectors changes the transmit covariance. The channel matrix can be represented as [21]

$$
\mathbf{H}_{D}=\mathbf{H}_{w} \mathbf{R}^{1 / 2}
$$

where $\mathbf{R}^{1 / 2}$ denotes the square root matrix of $\mathbf{R}$ [22] and $\mathbf{H}_{w}$ is the $(2 \times 2 M)$ spatially white complex Gaussian channel matrix [23]. Since the reference signal is obtained through 
a single transmit antenna of the MS, the eigen-mode beamforming is the same as the coherent beamforming. Letting $\mathbf{h}_{k}$ be the $k$ th row of the channel matrix $\mathbf{H}_{D}$, the beam weight $\mathbf{w}_{D}$ can be determined by

$$
\mathbf{w}_{D}=\sqrt{1+\frac{\alpha_{m^{\prime}}^{2}}{\alpha_{m}^{2}}} \frac{\mathbf{h}_{1}^{*}}{\left\|\mathbf{h}_{1}\right\|} .
$$

It can be seen that

$$
\mathbf{H}_{D} \mathbf{w}_{D}=\left[\begin{array}{c}
\mathbf{h}_{1} \\
\mathbf{h}_{2}
\end{array}\right] \mathbf{w}_{D}=\left[\begin{array}{c}
\sqrt{1+\alpha_{m^{\prime}}^{2} / \alpha_{m}^{2}}\left\|\mathbf{h}_{1}\right\| \\
\mathbf{h}_{2} \mathbf{w}_{D}
\end{array}\right]
$$

where

$$
\begin{aligned}
\mathbf{h}_{2} \mathbf{w}_{D} & =\left[\mathbf{H}_{w}\right]_{2} \mathbf{R}^{1 / 2} \mathbf{w}_{D} \\
& =\sqrt{1+\alpha_{m^{\prime}}^{2} / \alpha_{m}^{2}}\left[\mathbf{H}_{w}\right]_{2} \mathbf{R}^{1 / 2} \frac{\mathbf{R}^{* 1 / 2}\left[\mathbf{H}_{w}\right]_{1}^{*}}{\left\|\mathbf{h}_{1}\right\|} \\
& =\sqrt{1+\alpha_{m^{\prime}}^{2} / \alpha_{m}^{2}} \frac{\left[\mathbf{H}_{w}\right]_{2} \boldsymbol{\Sigma}^{2}\left[\mathbf{H}_{w}\right]_{1}^{*}}{\left\|\mathbf{h}_{1}\right\|}
\end{aligned}
$$

Here, $\left[\mathbf{H}_{w}\right]_{k}$ denotes the $k$ th row vector of $\mathbf{H}_{w}$, and it can be shown that

$$
\begin{aligned}
& E\left\{\| \frac{\left.\left[\mathbf{H}_{w}\right]_{2} \boldsymbol{\Sigma}^{2}\left[\mathbf{H}_{w}\right]_{1}^{*} \|^{2}\right\}}{\left\|\mathbf{h}_{1}\right\|}\right\} \\
& \approx \frac{E\left\{\left\|\left[\mathbf{H}_{w}\right]_{2} \boldsymbol{\Sigma}^{2}\left[\mathbf{H}_{w}\right]_{1}^{*}\right\|^{2}\right\}}{E\left\{\left\|\mathbf{h}_{1}\right\|^{2}\right\}} \\
& =\frac{\sum_{k=1}^{2 M} \lambda_{k}^{2}}{M\left(1+\alpha_{m^{\prime}}^{2} / \alpha_{m}^{2}\right)} .
\end{aligned}
$$

Thus, the effective channel of the second receive antenna can be rewritten as

$$
\mathbf{h}_{2} \mathbf{w}_{D}=\sqrt{1+\frac{\alpha_{m^{\prime}}^{2}}{\alpha_{m}^{2}}} \varphi h_{w}
$$

where $h_{w}$ denotes a zero-mean Gaussian random variable with unit variance. It can be seen that when the transmit antennas are highly correlated (i.e., $\lambda_{1} \gg \lambda_{2 M}$ ), the array gain of the second receive antenna is enhanced. The output SINR of an MMSEtype receiver can be represented by [9]

$$
\gamma=\alpha_{m}^{2}\left(\mathbf{H}_{D} \mathbf{w}_{D}\right)^{*} \mathbf{K}^{-1}\left(\mathbf{H}_{D} \mathbf{w}_{D}\right)
$$

where $\mathbf{K}$ denotes the covariance matrix of the interference plus noise, defined as

$$
\mathbf{K}=\sum_{i \in \Omega, \neq m, m^{\prime}} \alpha_{i}^{2}\left(\mathbf{H}_{i} \mathbf{w}_{i}\right)^{*}\left(\mathbf{H}_{i} \mathbf{w}_{i}\right)+N_{z} \mathbf{I}_{2} .
$$

\section{PERFORMANCE ANALYSIS}

\section{A. Output SINR of MMSE Receiver}

The output SINR of the MMSE receiver has a probability density function (pdf) represented as [24]

$$
p(\gamma)=\sum_{k=1}^{2} B_{k} \exp \left(-E\left\{\sigma_{k}\right\} \gamma\right)
$$

where

$$
B_{k}=\frac{\prod_{i=1}^{2} E\left\{\sigma_{i}\right\}}{\prod_{i=1, \neq k}^{2}\left(E\left\{\sigma_{i}\right\}-E\left\{\sigma_{k}\right\}\right)}
$$

and $\sigma_{k}$ denotes the eigenvalue of $\mathbf{K G}^{-1}$. Here, $\mathbf{G}$ denotes the covariance of the target signal, given by

$$
\begin{aligned}
\mathbf{G} & =\alpha_{m}^{2} E\left\{\left(\mathbf{H}_{D} \mathbf{w}_{D}\right)\left(\mathbf{H}_{D} \mathbf{w}_{D}\right)^{*}\right\} \\
& =\left(\sigma_{m}^{2}+\sigma_{m^{\prime}}^{2}\right)\left[\begin{array}{cc}
E\left\{\left\|\mathbf{h}_{1}\right\|^{2}\right\} & 0 \\
0 & \varphi^{2} E\left\{\left|h_{w}\right|^{2}\right\}
\end{array}\right] \\
& =\left(\sigma_{m}^{2}+\sigma_{m^{\prime}}^{2}\right)\left[\begin{array}{cc}
2 M & 0 \\
0 & \varphi^{2}
\end{array}\right] .
\end{aligned}
$$

It can be seen from (16) that the pdf of the output SINR can be determined by the mean eigenvalues of $\mathbf{K G}^{-1}$. Assuming that the major interference is from only sector $m^{\prime}$, the mean eigenvalues correspond to those of $N_{z} \mathbf{G}^{-1}$ since the inter-sector beamforming can avoid this major interference (i.e., $\mathbf{K}=N_{z} \mathbf{I}_{2}$ ). Thus, it can be shown that

$$
\begin{aligned}
& E\left\{\sigma_{1}\right\}=\frac{N_{z}}{2 M\left(\alpha_{m}^{2}+\alpha_{m^{\prime}}^{2}\right)}, \\
& E\left\{\sigma_{2}\right\}=\frac{N_{z}}{\varphi^{2}\left(\alpha_{m}^{2}+\alpha_{m^{\prime}}^{2}\right)} .
\end{aligned}
$$

When the interference comes from other cells, it is required to consider the covariance of the interference plus noise, $\mathbf{K}$. Since $\mathbf{w}_{i}$ can be assumed to be an $M$-dimensional unit-norm random vector and independent of $\mathbf{H}_{i}$, the received vector from sector $i$ can be represented as

$$
\mathbf{H}_{i} \mathbf{w}_{i}=\mathbf{h}_{w, i}
$$

where $\mathbf{h}_{w, i}$ is a $(2 \times 1)$ spatially white Gaussian channel vector [23] and

$$
E\left\{\mathbf{h}_{w, k}^{*} \mathbf{h}_{w, i}\right\}= \begin{cases}0, & k \neq i \\ 2, & k=i\end{cases}
$$

Thus, $\mathbf{K}$ can be rewritten as

$$
\mathbf{K}=\mathbf{C}_{I} \mathbf{P}_{I} \mathbf{C}_{I}^{*}+N_{z} \mathbf{I}_{2}
$$

In the presence of $L$ interferences from sectors $\left\{j_{k} \in \Omega ; \neq\right.$ $\left.m, m^{\prime}, k=1, \cdots, L\right\}, \mathbf{C}_{I}$ is a $(2 \times L)$ random matrix comprising $L$ interference vectors as columns, i.e.,

$$
\mathbf{C}_{I}=\left[\begin{array}{llll}
\mathbf{h}_{w, j_{1}} & \mathbf{h}_{w, j_{2}} & \cdots & \mathbf{h}_{w, j_{L}}
\end{array}\right]
$$

and $\mathbf{P}_{I}$ is an $(L \times L)$ diagonal matrix whose diagonal elements are the $\operatorname{RSS}\left\{\alpha_{j_{k}}^{2}\right\}$, given by

$$
\mathbf{P}_{I}=\left[\begin{array}{ccc}
\alpha_{j_{1}}^{2} & & \\
& \ddots & \\
& & \alpha_{j_{L}}^{2}
\end{array}\right] .
$$


It can be shown that the average output SINR of the MMSE receiver is approximated as (refer to Appendix)

$$
\begin{aligned}
E\{\gamma\} & =\int_{0}^{\infty} \gamma p(\gamma) \mathrm{d} \gamma \approx \frac{\sum_{k=1}^{2} E\left\{\sigma_{k}\right\}}{\prod_{k=1}^{2} E\left\{\sigma_{k}\right\}} \\
& =\frac{\sum_{i \in \Omega, \neq m, m^{\prime}} \alpha_{i}^{2}+N_{z}}{\prod_{k=1}^{2}\left(E\left\{\mu_{k}\right\}+N_{z}\right)} \cdot \frac{\operatorname{tr}\left[\mathbf{G}^{-1}\right]}{\operatorname{det}\left[\mathbf{G}^{-1}\right]} \\
& =\frac{\sum_{i \in \Omega, \neq m, m^{\prime}} \alpha_{i}^{2}+N_{z}}{\prod_{k=1}^{2} E\left\{\mu_{k}\right\}+N_{z} \sum_{k=1}^{2} E\left\{\mu_{k}\right\}+N_{z}^{2}} \cdot \frac{\operatorname{tr}\left[\mathbf{G}^{-1}\right]}{\operatorname{det}\left[\mathbf{G}^{-1}\right]}
\end{aligned}
$$

where $\operatorname{det}[\cdot]$ denotes the determinant of a matrix. It can further be approximated as (refer to Appendix)

$$
\begin{aligned}
E\{\gamma\} & =\frac{\left(A_{\text {sum }}+N_{z}\right) \cdot \gamma_{0}}{\left(E\left\{\beta_{1}\right\} E\left\{\beta_{2}\right\} / N_{z}\right) A_{\text {prod }}+2 A_{\text {sum }}+N_{z}} \\
& =\frac{\sum_{i \in \Omega, \neq m, m^{\prime}} \Gamma_{i}+1}{\left(E\left\{\beta_{1}\right\} E\left\{\beta_{2}\right\} / N_{z}^{2}\right) A_{\text {prod }}+2 \sum_{i \in \Omega, \neq m, m^{\prime}} \Gamma_{i}+1} \gamma_{0}
\end{aligned}
$$

where $A_{\text {sum }}=\sum_{i \in \Omega, \neq m, m^{\prime}} \alpha_{i}^{2}, A_{\text {prod }}=\prod_{i \in \Omega, \neq m, m^{\prime}} \alpha_{i}^{2}$, $\Gamma_{i}\left(=\alpha_{i}^{2} / N_{z}\right)$ denotes the average SNR of sector $i$, and $\gamma_{0}\left(=\operatorname{tr}\left[\mathbf{G}^{-1}\right] /\left(N_{z} \operatorname{det}\left[\mathbf{G}^{-1}\right]\right)\right)$ is the average SNR of the target signal. The first term of last expression in (26) represents the degradation due to the interference.

Consider the effect of the interference on users near the sector boundary in the inter-sector beamforming scheme. It can be assumed that users near the cell/sector boundary experience interference at most from two sources [26]. The use of the intersector beamforming can avoid interference from the adjacent sector, making users experience a single dominant interference (i.e., $\Gamma_{j_{1}} \gg 1$ ). Thus, the average output SINR of the MMSE receiver increases in proportion to the average SNR of the target signal at an expense of $3 \mathrm{~dB}$, i.e.,

$$
\begin{aligned}
E\{\gamma\} & \approx \frac{\Gamma_{j_{1}}+1}{\left(E\left\{\beta_{1}\right\} E\left\{\beta_{2}\right\} / N_{z}\right) \Gamma_{j_{1}}+2 \Gamma_{j_{1}}+1} \gamma_{0} \\
& \approx \frac{\Gamma_{j_{1}}}{2 \Gamma_{j_{1}}} \gamma_{0}=\frac{\gamma_{0}}{2}
\end{aligned}
$$

where $E\left\{\beta_{1}\right\}=2$ and $E\left\{\beta_{2}\right\}=0$ from Appendix. On the other hand, it can be shown that the use of the single-sector beamforming provides the average output SINR of the MMSE receiver given by

$$
\begin{aligned}
E\{\gamma\} & \approx \frac{\Gamma_{j_{1}}+\Gamma_{j_{1}}+1}{E\left\{\beta_{1}\right\} E\left\{\beta_{2}\right\} \Gamma_{j_{1}} \Gamma_{j_{2}}+2\left(\Gamma_{j_{1}}+\Gamma_{j_{2}}\right)+1} \gamma_{0} \\
& \approx \frac{1}{E\left\{\beta_{1}\right\} E\left\{\beta_{2}\right\}}\left(\frac{1}{\Gamma_{j_{1}}}+\frac{1}{\Gamma_{j_{2}}}\right) \gamma_{0}
\end{aligned}
$$

where $E\left\{\beta_{1}\right\}=3.5$ and $E\left\{\beta_{2}\right\}=0.5$ from Appendix. It can be seen that the average output SINR does not increase in proportion to the average SNR of the target signal, rather it significantly deteriorates due to the interference. Thus, the use of an inter-sector beamforming combined with the MMSE receiver improves the average output SINR by reducing the number of interferers. Since

$$
\operatorname{tr}\left[\mathbf{G}^{-1}\right]=\frac{\operatorname{tr}[\mathbf{G}]}{\operatorname{det}[\mathbf{G}]}=\frac{2 M+\varphi^{2}}{\left(\alpha_{m}^{2}+\alpha_{m^{\prime}}^{2}\right) 2 M \varphi^{2}}
$$

and

$$
\operatorname{det}\left[\mathbf{G}^{-1}\right]=\frac{1}{\left(\alpha_{m}^{2}+\alpha_{m^{\prime}}^{2}\right)^{2} 2 M \varphi^{2}},
$$

it can be shown that

$$
\gamma_{0}=\frac{\operatorname{tr}[\mathbf{G}]}{N_{z} \operatorname{det}[\mathbf{G}]}=\frac{\left(\alpha_{m}^{2}+\alpha_{m^{\prime}}^{2}\right)\left(2 M+\varphi^{2}\right)}{N_{z}} .
$$

When the neighboring sectors do not work in a cooperative mode, the inter-sector beamforming becomes a single-sector beamforming, allowing the adjacent sector to cause interference. This corresponds to the case when the first $M$ diagonal elements of $\boldsymbol{\Lambda}_{D}$ is one and the others are zero. Then, the covariance matrix $\mathbf{G}$ can be represented as

$$
\mathbf{G} \approx\left[\begin{array}{cc}
M & 0 \\
0 & \varphi^{2}
\end{array}\right]
$$

For the single-sector beamforming, it can be seen that

$$
\gamma_{0}=\frac{\operatorname{tr}[\mathbf{G}]}{N_{z} \operatorname{det}[\mathbf{G}]}=\frac{\alpha_{m}^{2}\left(M+\varphi^{2}\right)}{N_{z}} .
$$

Thus, the inter-sector beamforming outperforms the singlesector beamforming by achieving larger transmit array gain and power gain as well as interference mitigation.

\section{B. Mode Switching Criterion}

The inter-sector beamforming can provide noticeable transmit array gain and power gain to users near the sector boundary. However, it needs to utilize the resource two times compared to the single-sector beamforming. Thus, it may be desirable to properly switch between the inter-sector and the single-sector beamforming mode to maximize the output capacity as well as the output SINR. Define the achievable rate by [27]

$$
C(\gamma)=\log _{2}(1+\eta \gamma)
$$

where $\eta$ denotes the implementation loss (i.e., the gap between the Shannon capacity and practical scheme). Then, the intersector beamforming provides an output capacity $\tilde{C}_{I S}$ represented as

$$
\tilde{C}_{I S}(\gamma)=v C(\gamma)
$$

where $v$ denotes a weighting factor in a range of $0.5 \leq v \leq 1$. The case of $v=0.5$ indicates the penalty due to the use of double resource, whereas the case of $v=1$ indicates the consideration of achievable rate regardless of the used resource. The average achievable rate can be calculated as

$$
\begin{aligned}
E & \{C(\gamma)\}=\int_{0}^{\infty} C(\gamma) p(\gamma) d \gamma \\
= & \frac{E\left\{\sigma_{2}\right\} \exp \left\{E\left\{\sigma_{1}\right\} / \eta\right\} g_{1}-E\left\{\sigma_{1}\right\} \exp \left\{E\left\{\sigma_{2}\right\} / \eta\right\} g_{2}}{\left(E\left\{\sigma_{2}\right\}-E\left\{\sigma_{1}\right\}\right) \log 2}
\end{aligned}
$$

where $g_{k}$ denotes the exponential integral function, defined by $\int_{E\left\{\sigma_{k}\right\} / \eta}^{\infty} \frac{e^{-t}}{t} d t$. Thus, the mode switching criterion between 
the inter-sector and the single-sector beamforming can be determined as

$$
E\left\{\tilde{C}_{I S}\right\} \stackrel{\gtrless}{<}\left\{\tilde{C}_{S S}\right\}
$$

where $\tilde{C}_{S S}$ denotes the output capacity for the sing-sector beamforming, corresponding to the output capacity when $v=1$. The average output capacity depends on only $E\left\{\sigma_{1}\right\}$ and $E\left\{\sigma_{2}\right\}$ which can be obtained using long-term CSI such as the spatial correlation and the RSS of interfering sectors. Thus, the beamforming mode can analytically be switched based on the longterm CSI information.

\section{PERFORMANCE EVALUATION}

The analytic design and performance of the proposed beamforming scheme are verified by computer simulation. The proposed scheme is applied to a $(2 \times 2)$ MIMO configuration in correlated MIMO fading channel whose correlation matrix $[\boldsymbol{\Delta}(\rho)]$ is given by [28]

$$
[\boldsymbol{\Delta}(\rho)]_{p, q}=\rho^{|p-q|} \exp \left(j(p-q) \frac{\pi}{12}\right)
$$

where $\rho$ denotes the magnitude of the correlation coefficient between the two adjacent transmitter antennas, and $[\cdot]_{p, q}$ denotes the element of the $p$ th row and the $q$ th column. That is, $E\left\{\left[\begin{array}{ll}\mathbf{H}_{m} & \mathbf{H}_{m^{\prime}}\end{array}\right]^{*}\left[\begin{array}{ll}\mathbf{H}_{m} & \mathbf{H}_{m^{\prime}}\end{array}\right]\right\} / 2=\boldsymbol{\Delta}(\rho)$. It is assumed that the implementation loss is $5 \mathrm{~dB}$ and $v=0.5$. It is also assumed that sector 0 allocates the resource to the target user at each frame. The performance is evaluated in terms of the geometry defined by

$$
\begin{aligned}
G & =\frac{\alpha_{0}^{2}}{\sum_{i \in \Omega, \neq 0} \alpha_{i}^{2}+\sum_{i \notin \Omega} \alpha_{i}^{2}+N_{0}}=\frac{\alpha_{0}^{2}}{\sum_{i \in \Omega, \neq 0} \alpha_{i}^{2}+N_{z}} \\
& =\frac{\alpha_{0}^{2} / N_{z}}{\sum_{i \in \Omega, \neq 0} \alpha_{i}^{2} / N_{z}+1}=\frac{\Gamma_{0}}{\sum_{i \in \Omega, \neq 0} \Gamma_{i}+1} .
\end{aligned}
$$

In the following figures, the legend 'BF_MMSE,' 'BF_MRC,' 'ISBF_MMSE,' 'SHO_MMSE,' 'MD_MMSE,' 'FSS_MMSE,' and 'NullBF_MMSE' denote the single-sector beamforming with MMSE receiver, the single-sector beamforming with MRC, the inter-sector beamforming with MMSE receiver, the softer handover with MMSE receiver, the macro diversity handover with MMSE receiver, FSS with muting and MMSE receiver, and null beamforming with MMSE receiver, respectively. In the null beamforming, the serving sector and adjacent sector are assumed to transmit zero-forcing beam based on the CSI of the first receive antenna. The zero-forcing beam is generated at a loss of the transmit array gain, causing interference to the second receive antenna of the user serviced by the other sector.

Fig. 1 depicts the analytic and simulation results according to $\Gamma_{0}$, assuming interference only from adjacent sector 1 with the same RSS as the serving sector (i.e., $\Omega=\{0,1\}$ and $\Gamma_{1}=\Gamma_{0}$ ). In this case, the inter-sector beamforming can avoid the interference from the adjacent sector while increasing the transmit array gain and doubling the transmit power gain. It can be seen from Fig. 1(a) that the MMSE receiver increases the average output SINR in proportion to $\Gamma_{0}$, while the MRC does not mainly due to dominant interference. It can also be seen that the analytic

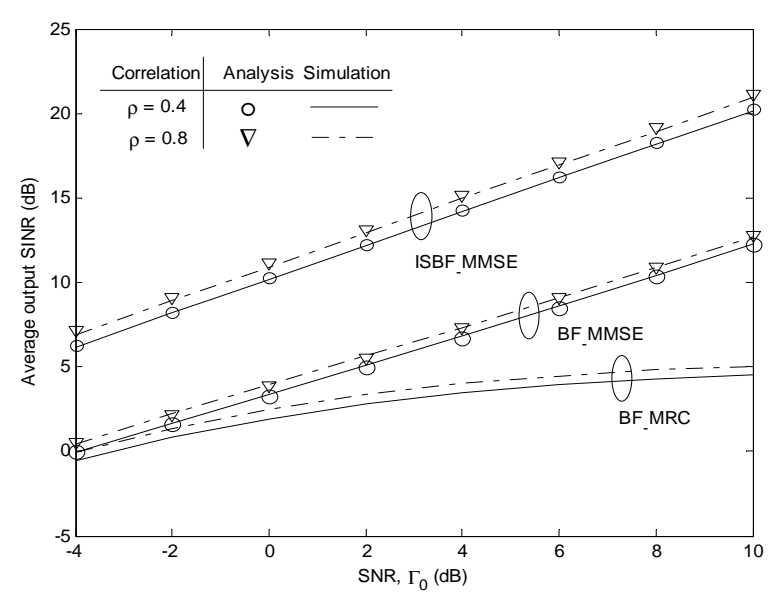

(a)

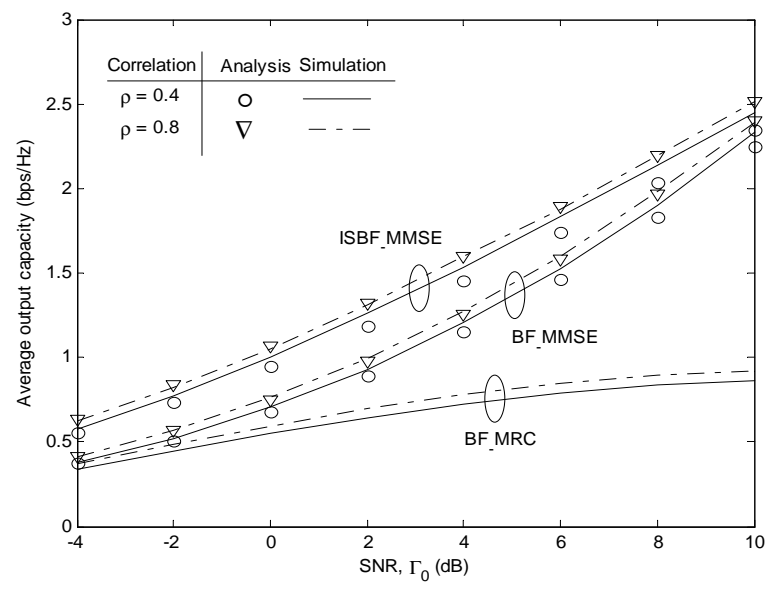

(b)

Fig. 1. Performance in the presence of interference from a single adjacent sector; (a) average output SINR (dB) and (b) average output capacity (bps/Hz).

results agree well with the simulation results. As $\rho$ increases, the performance improves due to the increase of the RSS of the second receiver antenna. It can be seen from Fig. 1(b) that the inter-sector beamforming scheme provides capacity higher than the other schemes when the SNR is not high. This is mainly because the capacity depends on the power gain in low SNR environments, while on the degree of freedom gain (i.e., the resource efficiency) in high SNR environments [10].

Fig. 2 depicts the analytic and simulation results according to $\Gamma_{0}$ in the presence of two interferences; one from adjacent sector 1 and the other from sector 2 (i.e., $\Omega=\{0,1,2\}$, and $\Gamma_{1}=\Gamma_{0}$ and $\Gamma_{1}=\Gamma_{0}+2 \mathrm{~dB}$ ). It can be seen that the MMSE receiver with the single-sector beamforming suffers from performance degradation due to unremoved interference, while the MMSE receiver with the inter-sector beamforming works well by properly removing the dominant interference. It can be seen that the average output SINR of the inter-sector beamforming increases in proportion to $\Gamma_{0}$ and that the capacity gap between the single-sector and the inter-sector beamforming increases as the SNR increases. 


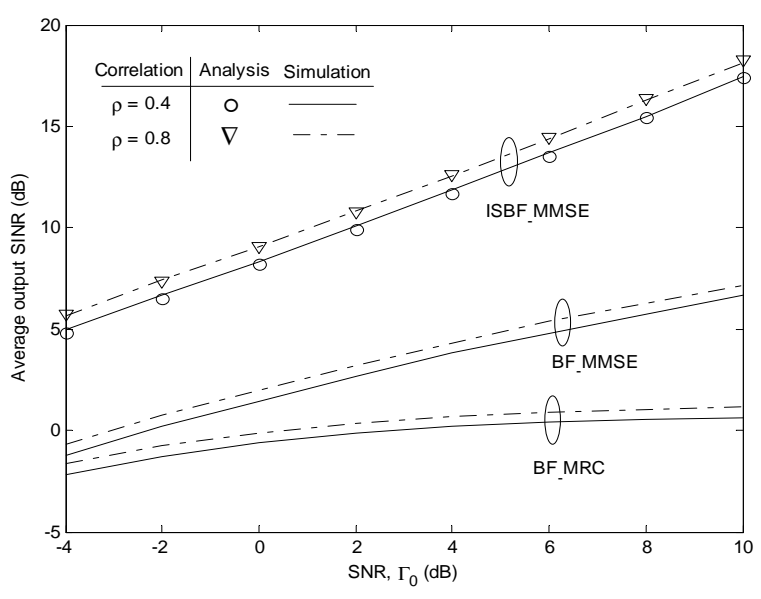

(a)

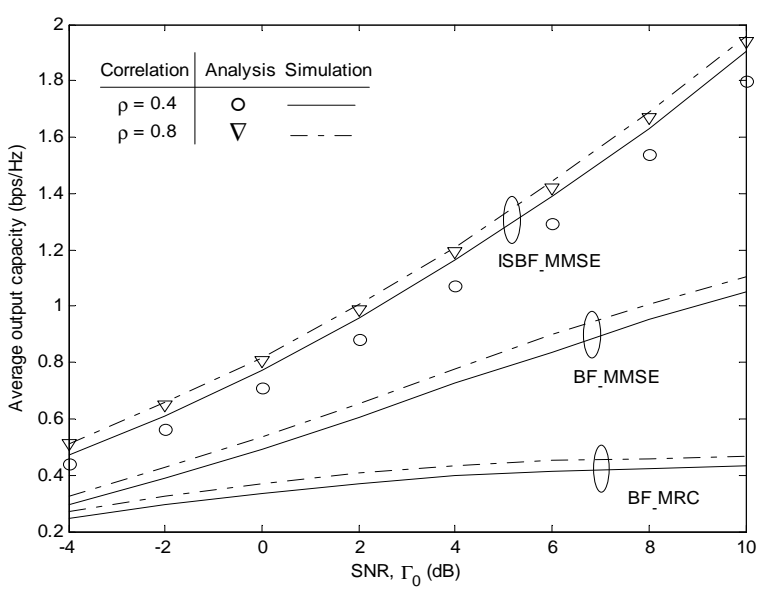

(b)

Fig. 2. Performance in the presence of interference from two adjacent sectors; (a) average output SINR (dB) and (b) average output capacity $(\mathrm{bps} / \mathrm{Hz})$.

Figs. 4 and 5 depict the performance in the same 19-cell environments (with $S=3$ ) as in Fig. 3, where the cell radius is 1 $\mathrm{km}$, the path loss follows $28.6+35 \log 10(d), d$ denotes the distance (in meters) between the sector and the user, and the sector antenna pattern follows $70^{\circ}$ ( $-3 \mathrm{~dB}$ beamwidth) with a frontto-back ratio of $20 \mathrm{~dB}$ [7]. It is assumed that sectors having an RSS larger than one half that of the serving sector belong to the active set (i.e., become an element of $\Omega$ with $\delta=-3 \mathrm{~dB}$ ), and that users are located near the boundary between sector 0 and 1 . Table 1 summarizes the active set and the corresponding SNR. It can be seen from Fig. 4(a) that the inter-sector beamforming outperforms the FSS with muting and the macro diversity handover mainly due to the transmit array gain through coherent transmission. It can also be seen that the null beamforming is not effective since it does not provide transmit array gain. The schemes cooperating two sectors are considered to halve the achievable rate since the two sectors transmit the same data signal. It can be seen from Fig. 4(b) that the single-sector beamforming provides capacity similar to or higher than the FSS with muting especially in the presence of a single dominant interfer-

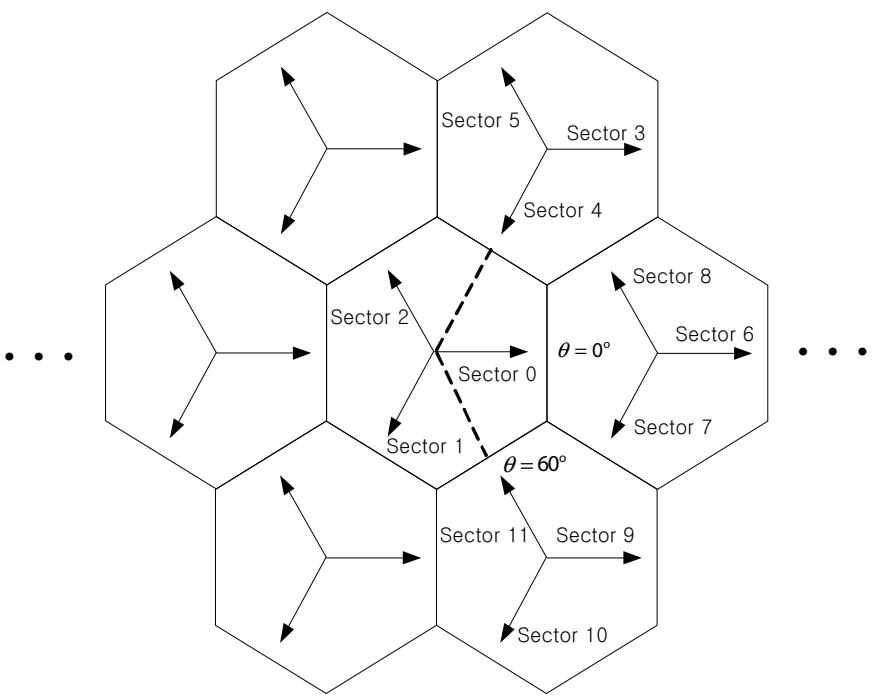

Fig. 3. 19 regularly placed cell environments (3 sectors per cell).

Table 1. The SNR of serving sector and interfering sectors near the sector boundary.

\begin{tabular}{lll}
\hline$G(\mathrm{~dB})$ & $\Omega$ & SNR $(\mathrm{dB})$ \\
\hline-0.5 & $\{0,1\}$ & $\Gamma_{0}=\Gamma_{1}=9.1$ \\
-1 & $\{0,1\}$ & $\Gamma_{0}=\Gamma_{1}=5.7$ \\
-2 & $\{0,1\}$ & $\Gamma_{0}=\Gamma_{1}=2.1$ \\
-4 & $\{0,1,11\}$ & $\Gamma_{0}=\Gamma_{1}=1.3, \Gamma_{11}=0.3$ \\
-5 & $\{0,1,11\}$ & $\Gamma_{0}=\Gamma_{1}=0.1, \Gamma_{11}=1.0$ \\
-7 & $\{0,1,11\}$ & $\Gamma_{0}=\Gamma_{1}=-1.8, \Gamma_{11}=2.2$ \\
\hline
\end{tabular}

ence. This is because the FSS with muting provides interference avoidance and power gain, but it does not provide transmit array gain. Since the null beamforming does not provide transmit array gain, it can yield performance worse than the single-sector beamforming. It can also be seen that the inter-sector beamforming outperforms the single-sector beamforming.

Fig. 5 depicts the performance of the beamforming schemes according to the number of transmit antennas when $\rho=0.8$. It can be seen that the average output SINR increases in proportion to the number of transmit antennas due to the transmit array gain, and that the capacity gap between the single-sector and the inter-sector beamforming decreases as the number of transmit antennas increases, implying the effectiveness of the inter-sector beamforming with a small number of transmit antennas.

Fig. 6 depicts the switching point between the single-sector and the inter-sector beamforming when users are located near the cell boundary at a direction of between $54^{\circ}$ and $60^{\circ}$ with respect to broadside of the sector array, and experience a geometry of $-5 \mathrm{~dB}$ at all directions. It can be seen from Table 2 that the RSS of the adjacent sector (i.e., sector 1) decreases as the user moves away from the sector boundary. In this case, the intersector beamforming can not sufficiently obtain transmit array gain and transmit power gain. It can also be seen that the switching point can accurately be determined. 


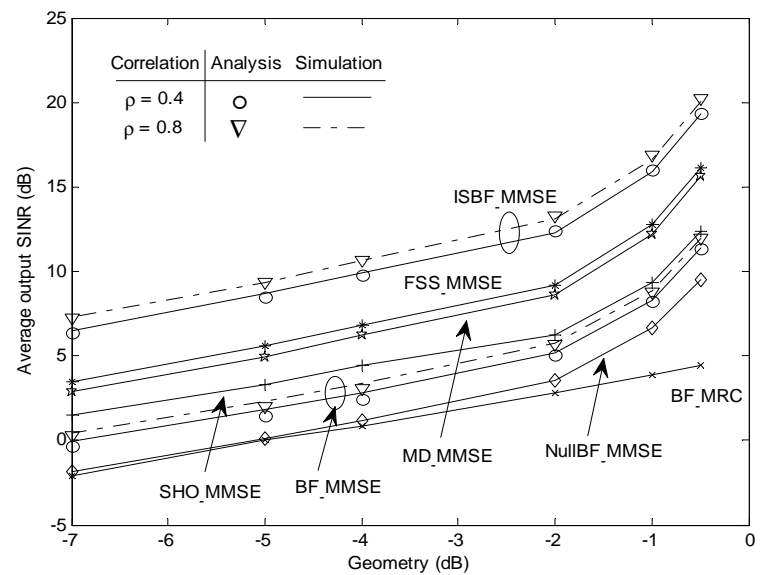

(a)

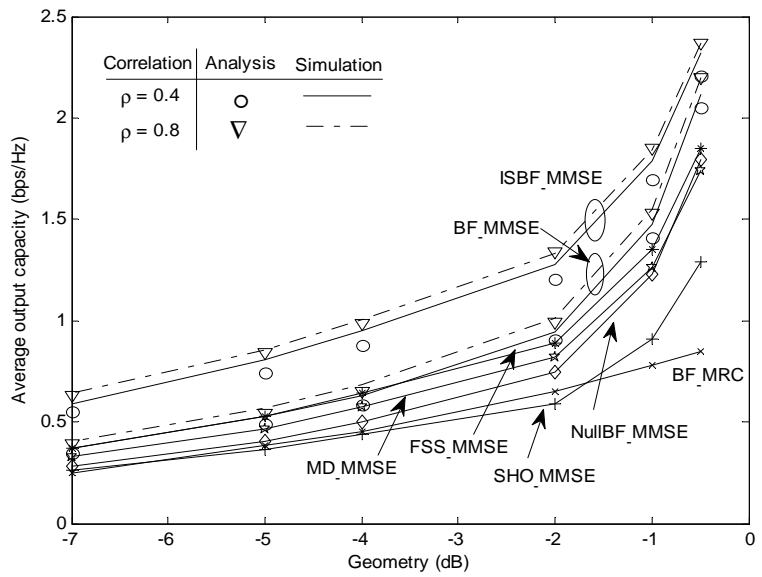

(b)

Fig. 4. Performance of users near the sector boundary; (a) average output SINR (dB) and (b) average output capacity (bps/Hz).

\section{CONCLUSION}

We have considered the use of inter-sector beamforming for the service of users near the sector boundary in TDD based cellular systems. The performance of the inter-sector beamforming has been analyzed in terms of the average output SINR and the capacity in correlated channel environments. The intersector beamforming mode can analytically be switched to the single-sector mode based on the long-term CSI. The simulation results show that the inter-sector beamforming is very effective for users near the sector boundary, outperforming conventional schemes such as the softer handover, macro diversity handover, FSS with muting, and null beamforming.

\section{APPENDIX}

In (23), $\mathbf{C}_{I}$ can be decomposed as [22]

$$
\mathbf{C}_{I}=\mathbf{U}_{I} \boldsymbol{\Sigma}_{I} \mathbf{V}_{I}^{*}
$$

where $\mathbf{U}_{I}$ and $\mathbf{V}_{I}$ are a $(2 \times 2)$ and $(L \times L)$ unitary matrix, respectively, and $\boldsymbol{\Sigma}_{I}$ is a $(2 \times L)$ diagonal matrix whose diag-

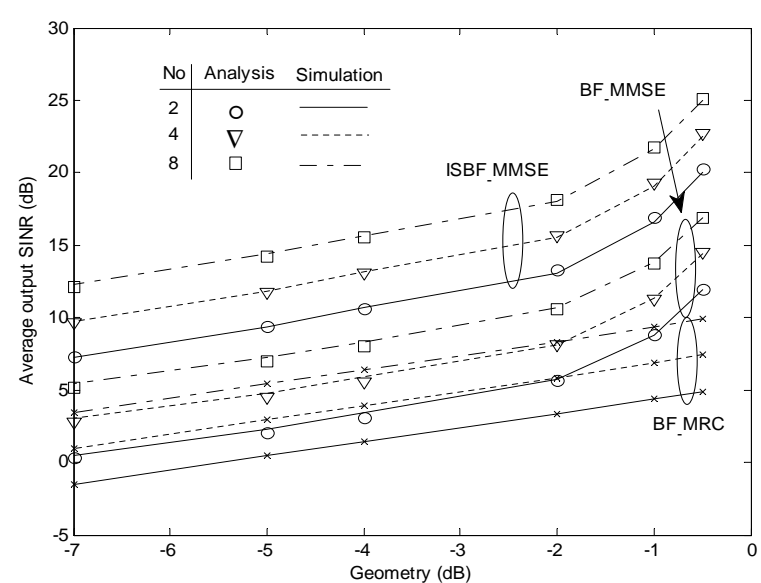

(a)

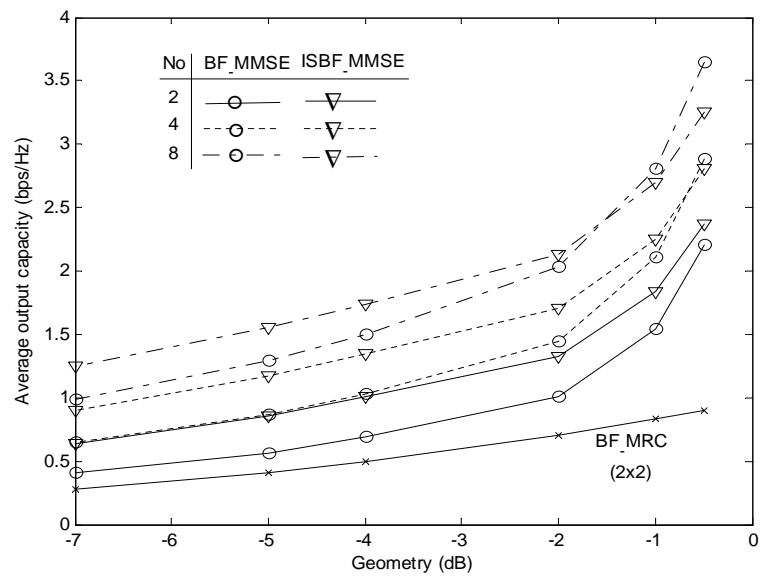

(b)

Fig. 5. Performance of the user near the sector boundary according to the number of transmit antennas; (a) average output SINR (dB) and (b) average output capacity (bps/Hz).

onal elements are $\sqrt{\beta_{1}}$ and $\sqrt{\beta_{2}}\left(\leq \sqrt{\beta_{1}}\right)$. Here, $\left\{\beta_{k}\right\}$ are the eigenvalues of $(2 \times 2) \mathbf{W}$ defined by [25]

$$
\mathbf{W} \triangleq \mathbf{C}_{I} \mathbf{C}_{I}^{*}=\mathbf{U}_{I} \boldsymbol{\Sigma}_{I}^{2} \mathbf{U}_{I}^{*}
$$

Since elements of $\mathbf{C}_{I}$ are complex Gaussian random variables with zero mean and unit variance, $\mathbf{W}$ is Hermitian matrix. It can be shown that [25]

$$
\begin{gathered}
E\left\{\beta_{1}\right\}=\frac{L\left(L^{2}+3\right)}{2^{L}}+\sum_{k=2}^{L-1} \frac{1}{2^{2 L-k-1}}\left(\begin{array}{c}
2 L-k-1 \\
L-2
\end{array}\right)\left(\begin{array}{c}
k \\
2
\end{array}\right), \\
E\left\{\beta_{2}\right\}=2 L-E\left\{\beta_{1}\right\}
\end{gathered}
$$

where $E\left\{\beta_{k}\right\}$ correspond to the mean eigenvalues of $\mathbf{W}$. Note that these values also correspond to the mean eigenvalues of covariance matrix of the interference when the interferers have unit power (i.e., $\mathbf{P}_{I}=\mathbf{I}_{L}$ ).

Let $\left\{\mu_{k}\right\}$ be the eigenvalues of $\mathbf{C}_{I} \mathbf{P}_{I} \mathbf{C}_{I}^{*}$ in (22) when the 
Table 2. The SNR of the serving sector and interfering sectors near the cell boundary at a geometry of $-5 \mathrm{~dB}$.

\begin{tabular}{lll}
\hline Direction $\left(^{\circ}\right)$ & $\Omega$ & SNR $(\mathrm{dB})$ \\
\hline 60 & $\{0,1,11\}$ & $\Gamma_{0}=0.1, \Gamma_{1}=0.1, \Gamma_{11}=1.0$ \\
58 & $\{0,1,11\}$ & $\Gamma_{0}=-0.1, \Gamma_{1}=-1.4, \Gamma_{11}=1.5$ \\
56 & $\{0,1,11\}$ & $\Gamma_{0}=-0.1, \Gamma_{1}=-2.8, \Gamma_{11}=2.0$ \\
54 & $\{0,11\}$ & $\Gamma_{0}=-1.6, \Gamma_{1}=-4.3, \Gamma_{11}=0.8$ \\
\hline
\end{tabular}

interferers have arbitrary power. It can be seen that

$$
\begin{aligned}
\sum_{k=1}^{2} E\left\{\mu_{k}\right\} & =E\left\{\sum_{k=1}^{2} \mu_{k}\right\}=E\left\{\operatorname{tr}\left[\mathbf{C}_{I} \mathbf{P}_{I} \mathbf{C}_{I}^{*}\right]\right\} \\
& =\sum_{i=1}^{L} \alpha_{j_{i}}^{2} E\left\{\left\|\mathbf{h}_{w, j_{i}}\right\|^{2}\right\}=2 \sum_{i=1}^{L} \alpha_{j_{i}}^{2} .
\end{aligned}
$$

Since [22]

$$
\begin{aligned}
\prod_{k=1}^{2} E\left\{\mu_{k}\right\} & =\operatorname{det}\left[\mathbf{C}_{I} \mathbf{P}_{I} \mathbf{C}_{I}^{*}\right] \\
& =\operatorname{det}\left[\mathbf{C}_{I}\right]^{2} \operatorname{det}\left[\mathbf{P}_{I}\right]=\left\{\prod_{k=1}^{2} \beta_{k}\right\} \operatorname{det}\left[\mathbf{P}_{I}\right]
\end{aligned}
$$

it can be approximated as

$$
\prod_{k=1}^{2} E\left\{\mu_{k}\right\} \approx\left\{\prod_{k=1}^{2} E\left\{\beta_{k}\right\}\right\} \operatorname{det}\left[\mathbf{P}_{I}\right] .
$$

Note that $\operatorname{det}\left[\mathbf{P}_{I}\right]$ is a constant. Thus, $E\left\{\mu_{k}\right\}$ can be calculated by finding a solution of

$$
x^{2}-x \sum_{k=1}^{2} E\left\{\mu_{k}\right\}+\prod_{k=1}^{2} E\left\{\mu_{k}\right\}=0,
$$

yielding

$$
\begin{aligned}
& E\left\{\mu_{1}\right\}, E\left\{\mu_{2}\right\} \\
& \quad \approx \sum_{i=1}^{L} \alpha_{j_{i}}^{2} \pm \sqrt{\left(\sum_{i=1}^{L} \alpha_{j_{i}}^{2}\right)^{2}-E\left\{\beta_{1}\right\} E\left\{\beta_{2}\right\} \mathbf{P}_{I} .}
\end{aligned}
$$

Similarly, it can be shown that

$$
\begin{aligned}
\sum_{k=1}^{2} E\left\{\sigma_{k}\right\} & =\operatorname{tr}\left[\left\{\mathbf{C}_{I} \mathbf{P}_{I} \mathbf{C}_{I}^{*}+N_{z} \mathbf{I}_{2}\right\} \mathbf{G}^{-1}\right] \\
& =\operatorname{tr}\left[\left\{\sum_{i=1}^{L} \alpha_{j_{i}}^{2} \mathbf{I}_{2}+N_{z} \mathbf{I}_{2}\right\} \mathbf{G}^{-1}\right] \\
& =\left\{\sum_{i=1}^{L} \alpha_{j_{i}}^{2}+N_{z}\right\} \operatorname{tr}\left[\mathbf{G}^{-1}\right] .
\end{aligned}
$$

Since [22]

$$
\begin{aligned}
\prod_{k=1}^{2} \sigma_{k} & =\operatorname{det}\left[\mathbf{K G}^{-1}\right] \\
& =\operatorname{det}\left[\mathbf{C}_{I} \mathbf{P}_{I} \mathbf{C}_{I}^{*}+N_{z} \mathbf{I}_{2}\right] \operatorname{det}\left[\mathbf{G}^{-1}\right] \\
& =\left\{\prod_{k=1}^{2}\left(\mu_{k}+N_{z}\right)\right\} \operatorname{det}\left[\mathbf{G}^{-1}\right]
\end{aligned}
$$



Fig. 6. Mode switching according to the direction of user near the cell boundary at a geometry of $-5 \mathrm{~dB}$.

it can be approximated as

$$
\prod_{k=1}^{2} E\left\{\sigma_{k}\right\} \approx\left\{\prod_{k=1}^{2}\left(E\left\{\mu_{k}\right\}+N_{z}\right)\right\} \operatorname{det}\left[\mathbf{G}^{-1}\right] \text {. }
$$

Note that $\operatorname{det}\left[\mathbf{G}^{-1}\right]$ is a constant. Similar to $E\left\{\mu_{k}\right\}, E\left\{\sigma_{k}\right\}$ can be calculated as

$$
E\left\{\sigma_{1}\right\}, E\left\{\sigma_{2}\right\}=\frac{1}{2}\left[B \mp \sqrt{B^{2}-4 C}\right]
$$

where $B=\left(\sum_{i=1}^{L} \alpha_{j_{i}}^{2}+N_{z}\right) \operatorname{tr}\left[\mathbf{G}^{-1}\right]$ and $C=\left\{\prod_{i=1}^{2}\right.$ $\left.\left(E\left\{\mu_{k}\right\}+N_{z}\right)\right\} \operatorname{det}\left[\mathbf{G}^{-1}\right]$.

\section{REFERENCES}

[1] Y. Xiang, J. Luo, and C. Hartmann, "Inter-cell interference mitigation through flexible resource reuse in OFDMA based communication networks," in Proc. EW, Apr. 2007.

[2] WiMAX Forum, "A comparative analysis of Mobile WiMAX deployment alternatives in the access network," May 2007.

[3] F. W. Vook, T. A. Thomas, and X. Zhuang, "Transmit diversity and transmit adaptive arrays for broadband mobile OFDM systems," in Proc. IEEE WCNC, vol. 1, Mar. 2003, pp. 44-49.

[4] F. Wang, A. Ghosh, C. Sankaran, and S. Benes, "WiMAX system performance with multiple transmit and multiple receive antennas," in Proc. IEEE VTC-spring, Apr. 2007, pp. 2807-2811.

[5] IEEE Std 802.16e, "Part 16: Air Interface for Fixed and Mobile Broadband Wireless Access Systems," Dec. 2005.

[6] WiMAX Forum, "Mobile WiMAX-Part I: A technical overview and performance evaluation," Feb. 2006.

[7] 3GPP TR 25.996, "3GPP technical specification group radio access network; Spatial channel model for MIMO simulations," V6.1.0, Dec. 2003.

[8] 3GPP TR 25.814, "3GPP technical specification group radio access network; Physical layer aspects for evolved UTRA," V6.1.0, Dec. 2006.

[9] J. H. Winters, "Optimum combining in digital mobile radio with cochannel interference," IEEE J. Sel. Areas Commun., vol. 33, pp. 523-539, Dec. 1997.

[10] D. Tse and P. Viswanath, Fundamentals of Wireless Communication. Cambridge University Press, 2005.

[11] R. Bachu, G. Song, K. Stewart, and X. Zhuang, "Linear interference cancellation for downlink map reception in IEEE 802.16e," in Proc. IEEE PIMRC, vol. 1, Sept. 2006, pp. 1-5. 
[12] S. Shamai and B. M. Zaidel, "Enhancing the cellular downlink capacity via co-processing at the transmitting end," in Proc. IEEE VTC-spring, vol. 3, May 2001, pp. 1745-1749.

[13] S. A. Jafar and G. J. Foschini, "PhantomNet: Exploring optimal multicellular multiple antenna systems," in Proc. IEEE VTC-fall, vol. 1, Sept. 2002, pp. 261-265.

[14] L. Shao and S. Roy, "Downlink multicell MIMO-OFDM: An architecture for next generation wireless networks," in Proc. IEEE WCNC, vol. 2, Mar. 2005, pp. 1120-1125.

[15] D. Dang, Z. Lin, and L. Chen, "Informative description of network architecture," IEEE C802.16g-05/005, IEEE 802.16 BWA working group, Jan. 2005.

[16] A. Morimoto, K. Higuchi, and M. Sawahashi, "Performance comparison between fast sector selection and simultaneous transmission with softcombining for intra-node B macro diversity in downlink OFDM radio access," in Proc. IEEE VTC-spring, vol. 1, 2006, pp. 157-161.

[17] J. Tan and G. L. Stuber, "Multicarrier delay diversity modulation for MIMO systems," IEEE Trans. Wireless Commun., vol. 3, pp. 1756-1763, Sept. 2004

[18] J. Tomcik, "QFDD and QTDD: Technology overview," IEEE C802.2005/68, IEEE 802.20 working group on MBWA, Oct. 2005.

[19] S. Plass, A. Dammann, and S. Kaiser, "Analysis of coded OFDMA in a downlink multi-cell scenario," in Proc. InOWo, Sept. 2004.

[20] G. H. Golub, and C. F. V. Loan, Matrix Computations. Johns Hopkins University Press, 1993

[21] P. Amers, E. Bonek, A. Burr, N. Czink, et al., "Survey of channel and radio propagation models for wireless MIMO systems," EURASIP J. Wireless Commun. Netw., vol. 2007, article ID 19070, Sept. 2007.

[22] G. Strang, Linear Algebra and Its Applications. Harcourt Brace Jovanovich College Publishers, 1988.

[23] A. Paulraj, R. Nabar, and D. Gore, Introduction to Space-Time Wireless Communications. Cambridge Universe Press, 2003.

[24] T. D. Pham and K. G. Balmain, "Multipath performance of adaptive antennas with multiple interferers and correlated fadings," IEEE Trans. Veh. Technol., vol. 48, pp. 342-352, Mar. 1999.

[25] M. Chiani, M. Z. Win, A. Zanella, R. K. Mallik, and J. H. Winters, "Bounds and approximations for optimum combining of signals in the presence of multiple cochannel interferers and thermal noise," IEEE Trans. Commun., vol. 51, pp. 296-307, Feb. 2003.

[26] 3GPP2, TSG-C, WG5 Evaluation AHG, "1xEV-DV evaluation methodology -Addendum (V6)," July 2001.

[27] J. Wang and T. S. Ng, Advances in $3 G$ Enhanced Technologies for Wireless Communications. Artech House, 2002.
[28] Q. T. Zang and X. W. Cui, "Outage probability for optimum combining of arbitrarily faded signals in the presence of correlated Rayleigh interferers," IEEE Trans. Veh. Technol., vol. 53, pp. 1043-1051, July 2004.

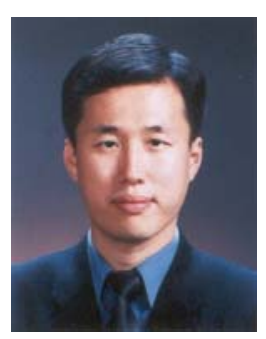

Jae-Heung Yeom received the B.S. and the M.S. degrees in electronics and communications engineering from Hanyang University, Seoul, Korea, in 1995 and 1997, respectively. He is now pursuing the Ph.D. degree in electrical engineering at Seoul National University (SNU). From 1997 to 2001, he was a research engineer at Samsung Electronics in Korea where he was involved in 3GPP standardization and development of cdma2000 modem. In 2002, he was a research engineer at LG Telecom in Korea. His research interin cellular systems, and OFDM systems. ests include MIMO systems, efficient MAC protocol

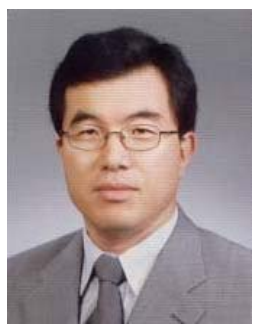

Yong-Hwan Lee received the B.S. degree from Seoul National University (SNU), Seoul, Korea, in 1977, the M.S. degree from the Korea Advanced Institute of Science and Technology (KAIST), Daejeon, in 1980, and the Ph.D. degree from the University of Massachusetts, Amherst, in 1989, all in electrical engineering. From 1980 to 1985, he was with the Korea Agency for Defense Development as a senior engineer. From 1989 to 1994, he worked for Motorola, Inc., where he was involved in the development of data transmission systems including high-speed modems. Since 1994, he has been with the School of Electrical Engineering, SNU, as a faculty member. He served as director of the Institute of New Media and Communications, SNU, from 2001 to 2003, and a vice-chair of the School of Electrical Engineering, SNU, from 2003 to 2005. He has also served international conferences including general chair for IEEE ISPLC2008, TPC vice-chair for IEEE ICC2005 and IEEE VTC 2003, and international journals as an editor. $\mathrm{He}$ has been working on the design of wireline/wireless transceivers, including digital subscriber loops, wireless local loops, wideband CDMA, and OFDM-based modems for next-generation mobile communications. 